\title{
Three Questions Confronted in Review Evaluation for Universities
}

\author{
Yaogang Li* \\ Shanghai, China \\ Feixiang Zhang \\ USST \\ Shanghai, China
}

Shanghai Education Evaluation Institute;

\author{
Yaocheng Luo \\ SPPC \\ Shanghai, China \\ Jingliang Chen \\ USST \\ Shanghai, China
}

\begin{abstract}
The paper mainly deals with three questions confronted in review evaluation for universities: how to understand review evaluation, how to implement selfevaluation, and how to prepare it. Scientific understanding of review evaluation is expounded in terms of evolution of evaluation models and the idea of "five degrees" (i.e. the key points of review evaluation); carrying out self-evaluation for colleges and universities is expanded from establishing factors, recruiting experts for self-evaluation and writing the self-evaluation report; external evaluation should be prepared with the common mind and ordinary attitude.
\end{abstract}

Keywords-review evaluation; five degrees; self-evaluation report; education

\section{INTRODUCTION}

In 2011, the Ministry of Education of the People's Republic of China (MOE) officially put forward the higher education evaluation system of "five into one". As an important part of evaluation of institutions, the review evaluation of the undergraduate teaching (hereinafter referred to as the "Review Evaluation") formally started in pilot institutions in 2013 and has been orderly carried out in colleges and universities all over the country. There are the most concerned questions for all colleges and universities: how to prepare for the review evaluation? Some thoughts and advice will be put forward and expanded from the following three aspects:

Have a scientific command of implementation requirements and ideas of review evaluation;

Place a high premium on self-evaluation of colleges and universities;

To meet external evaluation by experts with the common mind and ordinary attitude.

\section{HAVE A SCIENTIFIC COMMAND OF IMPLEMENTATION REQUIREMENTS AND IDEAS OF REVIEW EVALUATION}

\section{A. Fully understand implementation requirements of review} evaluation in terms of the evolution of evaluation modes

Education evaluation of institutions of higher education in China can be divided into three categories: qualified evaluation, level evaluation and review evaluation. In 1985, the State Education Commission (SEC) promulgated "Notice on Carrying out the Evaluation Research and Pilot of Higher Engineering Education”. Then, the pilot work of the evaluation on the educational level, specialties and courses of higher education has been launched in some provinces and cities.

In 1990, the first regulation on evaluation of higher education in China, "Provisional Regulations for Education Evaluation of Regular Institutions of Higher Education”, was promulgated by SEC. It further clarifies the nature, purposes, tasks, guidelines and basic forms of higher education evaluation. At the beginning of 1994, SEC began to organize a planned evaluation of the undergraduate teaching levels in ordinary colleges and universities. There are three kinds of evaluation: qualified evaluation, excellence evaluation and level evaluation at random. The qualified evaluation mainly aims at the newly-built colleges and universities. The excellence evaluation targets ones with a longer history, better foundation and higher teaching level. Level evaluation at random began in 1999, and it is mainly for ordinary colleges and universities between the two kinds mentioned above. The colleges and universities evaluated are randomly selected by MOE (SEC was renamed as MOE in 1998). In 2002, MOE combined the three evaluation schemes into one, which refers to the current one "Evaluation Scheme of Undergraduate Teaching Levels in Ordinary Colleges and Universities”. Since 2003, evaluation of undergraduate teaching levels in ordinary colleges and universities has been made and most of colleges and universities participate in it. The evaluation is concluded by four grades: excellent, good, qualified and unqualified[1]. In 2011, MOE ([2011]9) proposed establishing and improving the evaluation system of "Five into One", a teaching evaluation system which is compatible with the modern higher education system with Chinese characteristics. It is based on the self- 
evaluation of colleges and universities, mainly covering evaluation of colleges and universities (qualified evaluation or review evaluation), professional certification and evaluation, the international evaluation and constant monitoring of the basic teaching state data. It adopts the multi-evaluation model including government, universities, specialized agencies and the society[2]. The objects of qualified evaluation are newly built undergraduate universities that have not participated in the evaluation since 2000. The objects of review evaluation are ordinary undergraduate universities that have been approved in the evaluation. The "qualified evaluation", similar to the international "certification", serves to examine the educational qualifications in accordance with some standards. The "level evaluation", the so-called "grading model" (including excellent, good, qualified and unqualified), actually is to grade colleges and universities in terms of teaching levels. That is the level evaluation requires different standards of different levels rather than only the minimum standard required by qualified evaluation. There are unified standards all over the country for the qualified evaluation and level evaluation. However, review evaluation is different. It is made against goals set by universities themselves, and it has national reference but no unified evaluation standard. It has no graded conclusions but requires the realistic evaluation report. The basic features of review evaluation are: quality-oriented, classified evaluation, decentralization of power and keeping normal[3]. The emphasis of review evaluation is to guide colleges and universities to set up self-regulation mechanism, strengthen self-improvement and enhance educational levels and teaching quality.

\section{B. Have a deep understanding of the connotation of review evaluation in terms of "five degrees"}

The concept of review evaluation and its connotation should be understood. "Review" can be understood as the verification of the education "situation". The evaluation based on the review model is called review evaluation. Review evaluation is not a direct evaluation of education quality, but an indirect evaluation of education quality by evaluating the guarantee system of internal quality of universities. The focal points evaluated are the comprehensiveness, integrity, transparency and effectiveness of the guarantee mechanism of the internal quality of universities[4]. That is to say, with good and effective guarantee system, the education quality of colleges and universities can be guaranteed; otherwise not, even with good teachers, students and resources.

Review evaluation emphasizes on using one's own "ruler" to measure one's own "height", focusing on "five degrees"[5]: firstly, the degree of fulfillment of talent education effect and education goals; secondly, the degree of compatibility of education positioning, objectives and social needs; thirdly, the degree of guarantee of faculties and teaching resources for talent education; fourthly, the degree of effectiveness of operation of teaching quality guarantee system; fifthly, the degree of satisfaction of students and employers [6].

\section{Place a High Premium on Self-evaluation of UNIVERSITIES}

\section{A. Establish factors and key points of evaluation}

Review evaluation includes internal evaluation and external evaluation, both of which bear the same importance. Instead of drawing conclusions with different grades, review evaluation aims to elevate education level and teaching quality by guiding universities to set up self-regulation mechanism and keep making self-improvement according to the principle of “developing by evaluating”. In this way, self-evaluation gets more important in review evaluation in that it serves not only to "evaluate itself” but also to find out problems in self-evaluating to urge universities to develop and better themselves. Therefore, a comprehensive plan for evaluation should be made and all the faculty in colleges and universities should have a proper understanding that requirements and standards of review evaluation are set by themselves rather than by others. And colleges and universities should establish reviewed programs, factors and key points according to the specific situation. MOE proposes 24 reviewed factors and 64 reviewed key points and six reviewed programs plus a characteristic one, which usually refers to " $6+1$ " and covers all aspects of undergraduate teaching. But each college and university could make some changes and have its own reviewed factors and key points considering the specific situation by adding or reducing some. Self-evaluation in colleges and universities can start from evaluation of second-level schools or colleges or evaluation of majors. Sufficient preparation should be made before good organization of self- evaluation by experts. Self-evaluation experts play a very important role as their good summary and their constructive insight will have much influence on evaluation to a great degree.

\section{B. Invite self-evaluation experts}

The selection of self-evaluation experts should be considered both internally and externally. Review evaluation is a process of evaluation based on facts. Because each university should evaluate itself with its own "ruler" instead of the same standardized ruler for all universities, self-evaluation experts will be confronted with new challenges of keeping changing "ruler" to measure each university and of proper understanding of different situations of each university so as to make the effective evaluation by rightly using "right ruler". In fact, experts are required to not only employ the "ruler" of a university but also concern the positioning of the University “Accurate Measurement" and "right evaluation" in the review evaluation. Therefore, the selection and combination of experts bears the great importance. It is suggested that experts should be composed of peers and education management experts as the main part because self-evaluation should stick to the principle of combining internal and external experts. Besides, experts from the industry can be considered and even experts abroad can be invited to participate in the evaluation if necessary.

\section{Write self-evaluation report}

The quality of self-evaluation report directly reflects the result of review evaluation. To write self-evaluation actually is 
a process of organizing study, making self-reflection, understanding concepts, confirming positioning, spotting problems and making continuous improvement and so on. The effect of review evaluation will be directly affected by the depth of reflection, correctness of positioning, accuracy of problems, appropriateness of summarized characteristics and practicability of rectification plans. Instead of temporary report to deal with the evaluation, the report should be a historic document after repeated refinement, playing the role of a milestone for universities in their development in some period. It is not only a comprehensive conclusion and reflection of education and talent cultivation, but also a programmatic document used to direct universities to develop in years guided by its positioning and objectives.

The report should analyze the development, main problems, reasons, constructive ideas and corresponding measures of each work of universities one by one, sticking to the education positioning and development objectives of universities and taking "five degrees" as the logic line of evaluation. All of these are very important for the development of colleges and universities. Some questions should be paid attention to in writing the report:

1) Express advantages of the university with conciseness and conclusion with precision

To hunt down advantages of the university is the basis of upholding and inheriting them as well as the basis of positioning. Accurately summarized advantages can ensure the reasonable positioning. The evaluation of university education in self-evaluation report except cultivation plans and standards for courses is the evaluation standards set by the university itself, which is the basis of review evaluation for experts. Therefore, the university should put forward and express its advantages concisely and draw the clear conclusion with convincing facts based on the comprehensive but deep discussion among the second-level colleges and schools and all over the university, with no important achievements omitted, cheated and exaggerated and with no existing problems dodged and concealed.

2) Stick to the positioning of education and make a convincing report with facts

All the analysis of each program, factor and key point must stick to the positioning of education and educational objectives. That is, the positioning of education for top talents should be supported by corresponding steps, and the same is true for research-oriented talents and application-oriented talents. There should be basis for positioning, evidence for conclusion and data for analysis. Accurate words and proper ways must be employed to show the ideas of education and talent cultivation of the university. The ideas and positioning expressed in the report must be explained by facts. And all the conclusions must be based on conscientious, objective and comprehensive collection and summary of achievements of education. The effect of talent cultivation should be convincingly supported by lively cases, including typical ones with special effect and successful ones about students cultivation. Both analysis of problems and result of talent cultivation had better be backed up by data, which are more convincing. The whole report can't be tedious and its structure must be compact and precise by highlighting key points rather than touching every one. Of course, focal points should be highlighted based on deep understanding and good command of guidelines, key requirements and key points of inspection of review evaluation.

3) Find the crux and implement corresponding measures

In writing self-evaluation report of programs reviewed, each of three parts, problems, reasons and measures, is required to account for one third of the whole report. Crux must be found before correction measures are taken. Reasons must be analyzed thoroughly and measures must be feasible and vigorous. In analyzing reasons, both internal and external ones can be considered but the internal ones must be focused on. Neither can the report neither exaggerate existing problems nor ride off on side issues. Problems must be expressed certainly and clearly without ambiguity and evasiveness. The university can't copy problems of other universities and problems found should be ones which can be corrected and rectified and should not express in words like "should further do or improve. . . " because such an expression is not clear and specific enough for the university to correct and rectify problems. Whether achievements or problems, both of them should be supported by convincing data. Problems should be analyzed deep and thoroughly and the analysis of reasons should be accurate, specific and incisive and measures should be practical and feasible.

4) Put forward concise characteristics to show the heritage and development of the university

Characteristics should highlight the undergraduate teaching, both embodying the heritage of the history of universities and showing the leading role in development. Characteristics which can be applied to any university can't be considered ones for a university. The right characteristics for a university should be ones on which all the faculty of a university reach agreement after deep discussion. They should have "special features", and could be positioning and objectives, or highlights of education on teachers or students. All in all, characteristics, instead of slogans, should be the true reflection of the spirits, cultures and education features which have been developed in the university, should be the heritage of excellent education traditions and advanced education ideas and cultures.

5) Hold on to the requirement of "five degrees" throughout the report clearly and logically

Some relationships must be properly handled in writing the report with clear logic and consistency: the corresponding relationship between self-evaluation report and teaching basic state data and supporting materials; the relationship between meeting the requirement of reflecting reality and highlighting features; the relationship between concluding achievements and finding possible problems; the relationship between analyzing problems and addressing them. All in all, the report should be logically clear and thoroughly consistent with concise words and clear concepts. The achievements and problems should be described around the topic in the same way and be supported and analyzed both quantitatively and qualitatively with the consistent data. And "five degrees" should be the starting point of analyzing problems. 


\section{MeEt EXTERnal EVAluation by EXPerTs With COMMON Mind AND ORDinARY ATTITUdE}

Good self-evaluation can ensure a good external evaluation with the common mind and ordinary attitude. The first thing to be done for the university is to be well prepared ideologically, that is, all the faculty should both put high attention to the evaluation and keep the common mind and ordinary attitude without cheating, formalism and extravagance. The second thing is to ensure the preparation of accurate and complete materials. Usually, materials should be prepared and provided electronically in order to be convenient for experts to search for the relevant information. Therefore, these materials must be accurate because they are the basis of evaluation for the experts. Besides, state data must be in accordance with self-evaluation report with consistency and accuracy throughout the report. And the relevant supporting materials must be prepared as required. The third thing is the most important one in meeting the review evaluation. It is strongly suggested that the problems which have been found in self-evaluation should be solved before formal evaluation if possible. If problems have been found out, corrected, addressed in self-evaluation and listed out in the report, experts of external evaluation must be very satisfied.

\section{REFERENCES}

[1] Zhentian Liu. "Return to teaching life: a new round of college undergraduate teaching evaluation system design and its paradigm change in China " $[\mathrm{J}]$. Tsinghua University education research, vol. 6, pp. 39-45, 2013. (In Chinese)

[2] Zhiyi Li, Hong Zhu, Zhijun Liu. "How to correctly understand the review evaluation of undergraduate teaching"[J]. Chinese university teaching, vol. 10, pp. 4-8, 2012. (In Chinese)

[3] Binglin Zhong. "Following the rules, s moothly carry out the review evaluation of undergraduate teaching”[J]. Chinese higher education, vol. 6, pp. 4-7, 2014. (In Chinese)

[4] Yimin Yuan. "Review evaluation: how to truly understand and ensure a smooth evaluation" [J]. Development and evaluation of higher education, vol. 1, pp. 1-16, 2015. (In Chinese)

[5] Yan Wu. "Public governance of higher education and innovation of evaluation system of 'five one' " [J]. China higher education research, vol. 12, pp. 14-18, 2014. (In Chinese)

[6] Yuhao Cong. "Understanding and coping of a new round of review evaluation of undergraduate teaching” [J]. Evaluation of Chinese higher education, vol. 3, pp. 7-11, 2013. (In Chinese) 